\title{
Improvement of kink characteristic of proton implanted VCSEL with ITO overcoating
}

\author{
Fang-I Lai, Ya-Hsien Chang, Li-Hong, Laih, Hao-chung Kuo and S. C. Wang \\ Institute of Electro-Optical Engineering, \\ National Chiao Tung University, Hsinchu, Taiwan, R.O.C.
}

\begin{abstract}
Proton implanted VCSEL has been demonstrated with good reliability and decent modulation speed up to $1.25 \mathrm{~Gb} / \mathrm{s}$. However, kinks in current vs light output (L-I) has been always an issue in the gain-guided proton implant VCSEL. The kink related jitter and noise performance made it difficult to meet $2.5 \mathrm{~Gb} / \mathrm{s}(\mathrm{OC}-48)$ requirement. The kinks in L-I curve can be attributed to non-uniform carrier distribution induced non-uniform gain distribution within emission area. In this paper, the effects of a Ti/ITO transparent over-coating on the proton-implanted AlGaAs/GaAs VCSELs (15um diameter aperture) are investigated. The kinks distribution in L-I characteristics from a 2 inch wafer is greatly improved compared to conventional process. These VCSELs exhibit nearly kink-free L-I output performance with threshold currents $\sim 3 \mathrm{~mA}$, and the slope efficiencies $\sim 0.25$ W/A. The near-field emission patterns suggest the Ti/ITO over-coating facilitates the current spreading and uniform carrier distribution of the top VCSEL contact thus enhancing the laser performance. Finally, we performed high speed modulation measurement. The eye diagram of proton-implanted VCSELs with Ti/ITO transparent over-coating operating at $2.125 \mathrm{~Gb} / \mathrm{s}$ with $10 \mathrm{~mA}$ bias and $9 \mathrm{~dB}$ extinction ratio shows very clean eye with jitter less than 35 ps.
\end{abstract}

KEYWORDS: VCSEL, Proton implant, ITO, Kink, kink improvement, High-speed operation, uniform carrier distribution

\section{INTRODUCTION}

The vertical cavity surface emitting lasers (VCSELs) have generated research and commercial interests worldwide, because VCSELs possess several unique features over the edge emitting lasers. The VCSELs emit circular emission beam with low divergence, making optical fiber coupling efficient and easier than the edge

Vertical-Cavity Surface-Emitting Lasers VIII, edited by Chun Lei,

Kent D. Choquette, Sean P. Kilcoyne, Proceedings of SPIE Vol. 5364

(SPIE, Bellingham, WA, 2004) $\cdot 0277-786$ X/04/\$15 doi: 10.1117/12.530023 
emitting Lasers. The VCSELs can be tested on wafer level before packaging, and can be easily fabricated into dense 2-dimentional laser arrays. Currently, there are two main categories of VCSELs namely oxide-confined VCSELs and proton implanted VCSELs. The oxide confined VCSELs have an oxided boundary surrounding the emitting aperture for index-guiding and current confinement resulting well defined transverse modes at low bias current [1, 2]. However, the oxide VCSELs have a few technical issues. The oxidation procedure depends strongly on materials and processing parameters which could change during the process making control of the aperture size relatively difficult. In addition, the strain and defects introduced by the oxidation process have shown to cause reliability problem [3]. Compared to the oxide-confined VCSELs, the proton implanted VCSELs have a relatively simple fabrication process and have been demonstrated with good reliability [4]. However, due to the gain-guided nature of the proton implanted VCSELs, the kink in light output power versus current (L-I) has been an issue [5], and the laser power output jitter and noise also tend to limit the modulated speed around $1.25 \mathrm{~Gb} / \mathrm{s}$ [4] that hardly meets the 2.5 $\mathrm{Gb} / \mathrm{s}$ (OC-48) requirement. Earlier reports on edge emitting lasers related kinks in L-I curve to the spatial hole-burning [6], mode hopping [7] and mode jumps due to an increase in the injection current [8]. Therefore, the frequency of lasing mode could affected by the fluctuation and non-uniformity in both junction temperature and injected carrier density [9]. For the VCSELs, the reports also indicated the transverse mode structures depend on the drive current,[10] and the injection current from the periphery of the top contact aperture into the active region tends to cause current crowding and non-uniform current spreading [11-14]. Such non-uniform current spreading might lead to the kinks in L-I curve. Therefore the improvement of current spreading and reduction of current in VCSELs could reduce the kinks and thus improve the device modulation speed.

Recently there were reports using of transparent indium-tin-oxide (ITO) to replacing regular Ti/Au or Ti/Pt/Au p-contact of the VCSELs and showed that the ITO has good ohmic contact similar to the regular contact and increase contact transparency. These include the use of ITO [15, 16], Au-plated ITO [17] as the top contact and ITO as top ring contact [18]. However, the effect of these new contact scheme on the VCSEL performance such as kink characteristics of L-I curve, and modulation response were not clearly mentioned. In this paper, we report results of incorporation of a new p-contact scheme using Ti and ITO transparent overcoating on the regular p-contact of the implanted VCSEL that show substantial improvement in the kink characteristics in L-I curve, and the modulation response of the VCSEL.

\section{EXPERIMENT}

The GaAs VCSEL wafer structure was grown on the $n+-G a A s$ substrate by a metal organic chemical vapor deposition system. The bottom DBR consists of a 30.5-period n-doped quarter-wave stack of Al0.12Ga0.88As/AlAs and the top DBR consists of 19.5-period p-doped Al0.12Ga0.88As/AlAs. The graded-index separate-confinement heterostructure active region has an undoped three-quantum-well (3QWs) GaAs/Al0.3Ga0.7As, a lower 
linearly-graded undoped AlxGa1-xAs $(\mathrm{x}=0.6 \rightarrow 0.3)$ waveguide layer and an upper linearly-graded undoped-AlxGa1-xAs $(\mathrm{x}=0.3 \rightarrow 0.6)$ waveguide layer. A heavily $\mathrm{C}$-doped $\mathrm{p}$-type GaAs cap layer was grown to facilitate p-ohmic contact. The VCSELs were fabricated using a planar process. First, the VCSEL wafer was cleaned by acetone, isopropyl alcohol and D.I. water. Then a regular p-contact metallization of Ti (200 ̊) /Au (1500 ̊) were deposited by E-beam evaporator with $15 \mu \mathrm{m}$ diameter emission aperture and a contact pad size of $350 \times 350 \mu \mathrm{m} 2$. Then a Ge $(200 \AA)$ / Au (400 ̊) / Ni (140 ̊) / Au (1500 ̊) layer was deposited as the n-ohmic contact. The device was annealed in a rapid thermal annealing system at $380 \mathrm{oC}$ under $\mathrm{N} 2$ ambient for 30sec. The device was then subjected to the proton implantation with an energy of $200 \mathrm{KeV}$ and a dosage of $1 \times 1015 \mathrm{~cm}-2$ with an aperture of $20 \mu \mathrm{m}$ diameter. All the VCSELs were tested prior to the overcoating. The tested VCSELs were then overcoated with Ti/ITO transparent film on top of the regular Ti/Au p-contact by RF sputtering. The thickness of Ti is $40 \AA$ and the thickness of ITO (In2O3-SnO2) is $3200 \AA$. The thin Ti film was chosen because of its high transparency ( 92\% at $850 \mathrm{~nm}$ ), low annealing temperature and good adhesive property and ohmic contact with GaAs. Subsequently, the device was annealed at $380 \mathrm{oC}$ under N2 ambient for $30 \mathrm{sec}$ to form ohmic contact. The dimension of the transparent overcoating is $300 \times 300 \mu \mathrm{m} 2$ slightly smaller than the regular p-contact pad size for easy probing. The schematic of the fabricated VCSEL device is shown in Fig. 1.

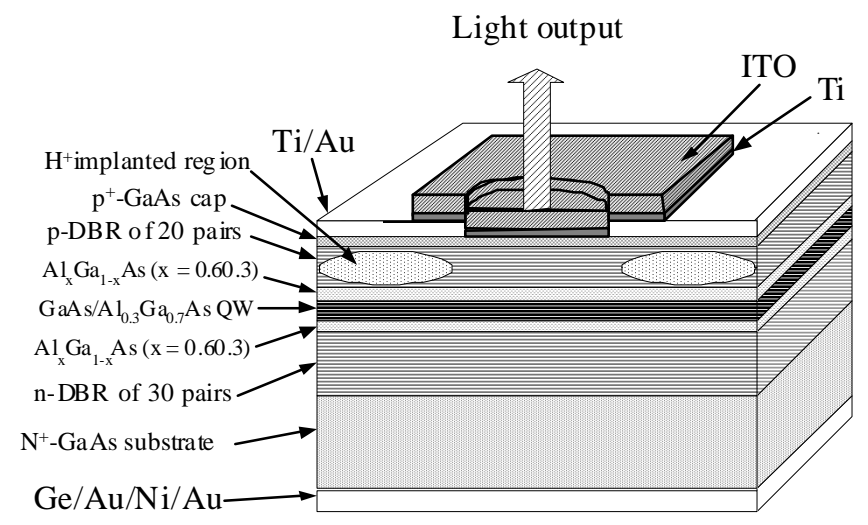

Figure 1. The schematic of the overcoated VCSEL device.

\section{RESULTS AND DISCUSSION}

Figs. 2(a), 2(b), and 2(c) show the comparisons of the L-I-V characteristics of three adjacent VCSELs before and after the Ti/ITO overcoating. The output power of VCSELs show almost the same after transparent overcoating, and all VCSELs only have slightly change in series resistance from $33 \Omega$ to $38 \Omega$ after Ti/ITO overcoating which 
consist with the previous reports $[16,18]$. The L-I curves of the overcoated VCSELs show a substantial improvement in the kinks compared with the uncoated VCSELs. To quantify the kink characteristics in L-I curve, we define a Derivative Kink Factor (DKF) which measure the mean square deviation of the local slope from the average slope, normalized to the average slope of the L-I cure as show below:

$$
\text { Derivative Kink Factor }=\left.\frac{\left.\left\langle(\partial L / \partial I-\langle\partial L / \partial I\rangle)^{2}\right\rangle^{1 / 2}\right|^{L \max / 2}}{\langle\partial L / \partial I\rangle}\right|_{L_{I_{h h}}}
$$

Where $\mathrm{L}$ is the light output power, $\mathrm{I}$ is the driving current, Lmax is the maximum light output power, and LIth is the light output power at the threshold. To minimize the effect of thermal rollover at higher power output, the DKF is only evaluated from threshold to Lmax/2. Table 1 shows the calculated values of DKF of these three devices for current range from the threshold to $15 \mathrm{~mA}$. The data indicate the DKF values of all these devices with overcoating reduce. In particular, the device1 and device 3 show 3 to 6 time reduction while the device 2 also shows $24 \%$ reduction in DKF value. From the L-I curves and the estimated DKF values, the Ti/ITO overcoating clearly improves the kink characteristics of the implanted VCSEL.

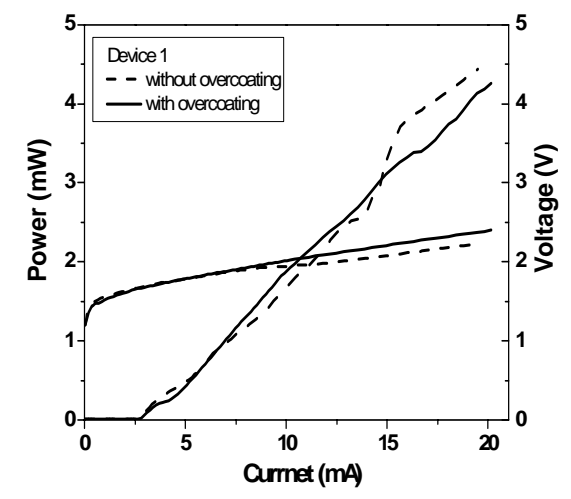

( a )

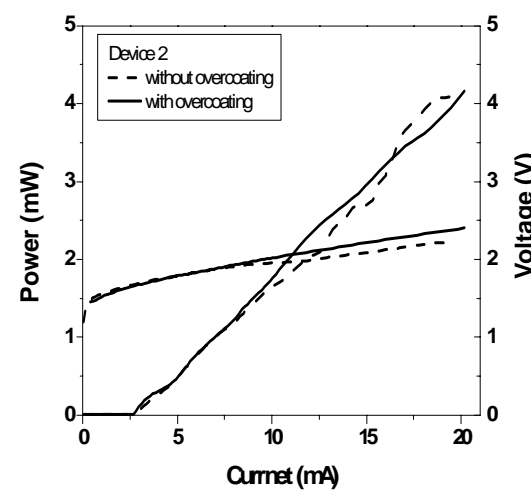

(b)

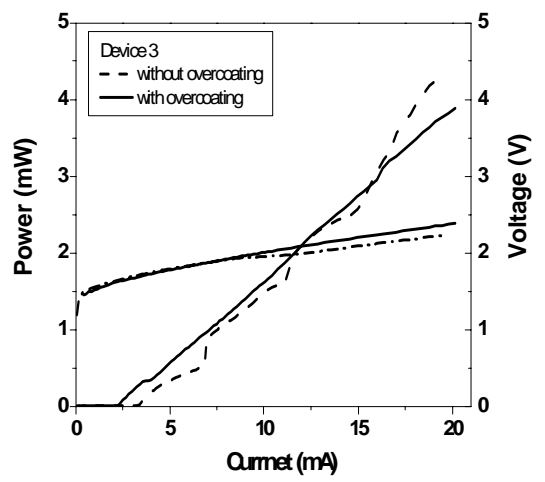

(c)

Figure 2. The L-I-V characteristics of the VCSELs with and without Ti/ITO transparent overcoating : (a) device1, (b) device2 and (c) device3. 
Table 1. The derivative kink factors of three devices with and without Ti/ITO transparent overcoating.

\begin{tabular}{|c|c|c|c|}
\hline & $\begin{array}{c}\text { Device } \\
1\end{array}$ & $\begin{array}{c}\text { Device } \\
2\end{array}$ & Device 3 \\
\hline $\begin{array}{c}\text { Without } \\
\text { Ti/ITO }\end{array}$ & 0.62 & 0.26 & 1.05 \\
\hline $\begin{array}{c}\text { With } \\
\text { Ti/ITO }\end{array}$ & 0.23 & 0.21 & 0.17 \\
\hline
\end{tabular}

The device 3 then was measured the eye diagram before and after Ti/ITO overcoating respectively operating at $2.125 \mathrm{~Gb} / \mathrm{s}$ with $10 \mathrm{~mA}$ bias and $9 \mathrm{~dB}$ extinction ratio. The uncoated VCSEL failed to pass the 2.125 Gb/s Eye-Mask and has a large jitter over 40ps. While the overcoated VCSEL showed a wide open eye pattern, and easily passed the $2.125 \mathrm{~Gb} / \mathrm{s}$ with a jitter of less than $35 \mathrm{ps}$. The result indicates the Ti/ITO overcoated VCSEL with lower DKF values has substantially improved in the high-speed performance characteristic.

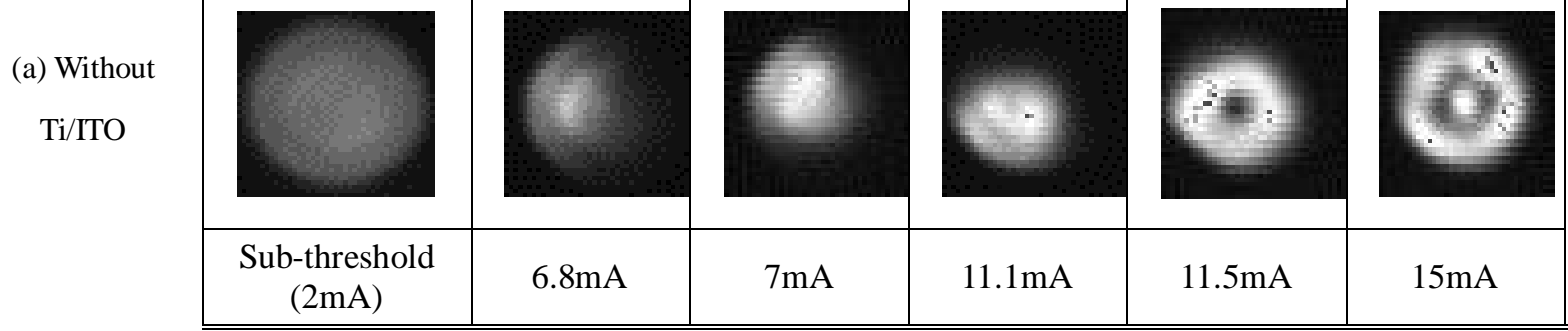

(b) With

Ti/ITO

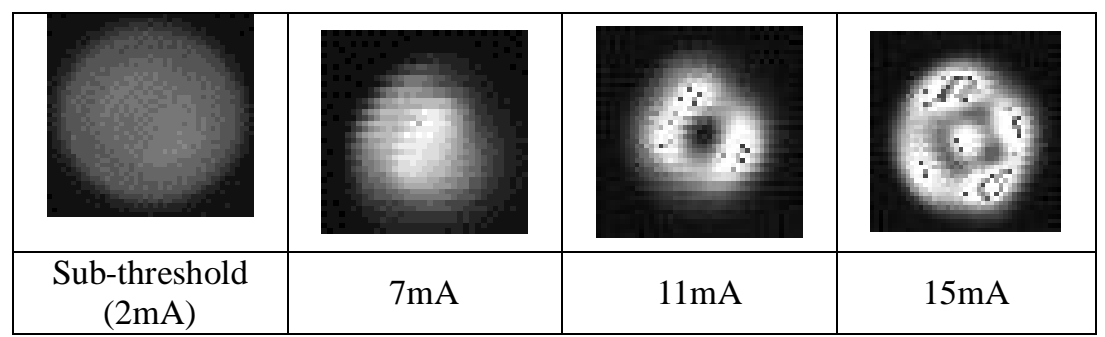

Figure 3. The near-filed emission patterns of the VCSEL device 3 (a) without and (b) with Ti/ITO transparent overcoating at different injecting currents

The improvement in the kink characteristics and modulation response of the Ti/ITO overcoated implanted VCSEL maybe the result of better current injection and spread induced by the overcoating. Since the change of light 
emission pattern correspond to the change of current injection density [6] into the VCSELs, Fig. 3(a) and 3(b) compare the near-field optical microscope images of the emission patterns of the VCSEL before and after overcoating in the currents which the kinks take placed. Fig 3(a) clear shows different lasing mode patterns when kink take place in L-I curve, and Fig 3(b) shows that the overcoated VCSELs have more uniform and stable emission patterns at the same current level than the uncoated devices. The results seem to suggest that the overcoated VCSELs allow better uniform current injection than the uncoated devices resulting in better emission mode stability which could lead to the improvement in the kink characteristics and high speed response.

\section{CONCLUSION}

In summary, we demonstrated the enhancement in the performance of proton-implanted VCSEL by using a Ti/ITO overcoating on the regular p-contact. The kink characteristics of the VCSEL with the Ti/ITO overcoating show substantial improvement with as large as six fold reduction in the derivative kink factor. The high-speed response of the overcoated device shows better performance than the uncoated devices with clear eye with 35ps jitter operating at $2.125 \mathrm{~Gb} / \mathrm{s}$ with $10 \mathrm{~mA}$ bias and $9 \mathrm{~dB}$ extinction ratio. These improvements in the implanted VCSEL performance would be due to the better current spreading and uniformity induced by the overcoating. This overcoating technique should be applicable to the other types of VCSELs.

\section{ACKNOWLEDGEMENT}

This work was supported in part by the National Science Council of Republic of China (ROC) in Taiwan under contract No. NSC91-2215-E009-030 and by the Academic Excellence Program of the ROC Ministry of Education under the contract No. 88-FA06-AB. The authors would like to thank Dr. C. H. Lin of Agilent Techology, Drs. C. P. Kuo and G. Hasnain of LuxNet corporation and C. P. Sung of ITRI for technical discussion.

\section{REFERENCES}

[1] C.W. Wilmsen, H. Temkin, and L.A. Coldren, eds., Vertical-Cavity Surface-Emitting Lasers: Design, Fabrication, Characterization, and Applications. Cambridge University Press, 1999.J.

[2] K. L. Lear, A. Mai, K. D. Choquette, S. P. Kilcoyne, R. P. Schneider, Jr, and K. M. Geib. "High-frequency modulation of oxide-confined vertical cavity surface emitting lasers," Electron Lett. 32, pp. 457, 1996

[3] K. L. Lear, S.P. Kilcoyne, R.P. Schneider, and J.A. Nevers. "Life-testing oxide confined VCSELs: too good to last?" Fabrication, Testing, and Reliability of Semiconductor Lasers, M. Fallahi and S.C. Wang, eds., Proceedings of the SPIE 1996; 2683:114-122.

[4] A. Tatum, A. Clark, J. K. Guenter, R. A. Hawthorne, and R. H. Johnson. Commercialization of Honeywell's 
VCSEL technology. Vertical-Cavity Surface-Emitting Lasers IV, K. D. Choquette and C. Lei, editors, Proceedings of the SPIE 2000;3946:2-13.

[5] K. D. Choquette, S.P. Kilcoyne, K.L. Lear. R. P. Schneider. "Index guiding dependent Effects in Implant and oxide confined Vertical-cavity Lasers” IEEE Photonics Technology Lett. 8, pp. 740, 1996.

[6] Roy Lang. "Lateral transverse mode instability and its stabilization in stripe geometry injection lasers," IEEE J. Quantum Electronics QE-15, pp. 718, 1979.

[7] Tien-Pei Lee, Charles A. Burrus, JR.., Pao-Lo Liu, William B. Sessa, and Ralph A. Logan. "An investigation of the frequency stability and temperature characteristics of $1.5 \mu \mathrm{m}$ coupled-cavity injection lasers," IEEE J. Quantum Electronics QE-20, pp. 374, 1984.

[8] G. R. Gray and R. Roy. "Bistability and mode hopping in a semiconductor laser," J. Opt. Soc. Am. B 8 pp. 632 , 1991.

[9] Y. Liu, W. C. Ng, B. Klein and K. Hess. "Effects of the spatial nonuniformity of optical transverse modes on the modulation response of vertical-cavity surface-emitting lasers,” IEEE J. Quantum Electronics 39, pp. 99, 2003.

[10] C. J. Chang-Hasnain, J. P. Harbison, G. Hasnian, A. C. Von Lehmen, L. T. Florez and N. G. Stoffet. ”Dynamic polarization, and transverse mode characteristics of vertical cavity surface emitting lasers," IEEE J. Quantum Electronics 27 (1991) 1402.

[11] N. K. Dutta. "Analysis of current spreading, carrier diffusion, and transverse mode guiding in surface emitting lasers,” J. Appl. Phys. 68, pp. 1961, 1990.

[12] W. Nakwaski and M. Osinski. "Thermal properties of etched-well surface-emitting semiconductor lasers," IEEE J. Quantum Electronics 27, pp. 1391, 1991.

[13] H. Wada, D. I. Babic, M. Ishikawa, and J. E. Bowers. "Effects of nonuniform current injection in GaInAsP/InP vertical-cavity lasers,” Appl. Phys. Lett. 60, pp. 2974, 1992.

[14] S. Sekiguchi, T. Miyamoto, T. Kimura, G. Okazaki, F. Koyama, and K. Iga. "Improvement of current injection uniformity and device resistance in long-wavelength vertical-cavity surface-emitting laser using a tunnel junction,” Jap. J. Appl. Phys. 39, pp. 3997, 2000.

[15] M. A. Matin, A. F. Jezierski, S. A. Bashar, D. E. Lacklison, T. M. Benson, T. S. Cheng, J. S. Robers, T. E. Sale, J. W. Orton, C. T. Foxon, and A. A. Rezazadeh." Optically transparent indium-tin-oxide (ITO) ohmic contacts in the fabrication of vertical-cavity surface-emitting lasers," Electron. Lett. 30, pp. 318, 1994.

[16] C. L. Chua, R. L. Tornton, D. W. Treat, V. K. Yang, and C. C. Dunnrowicz. "Indium tin oxide transparent electrodes for broad-area top-emitting vertical-cavity lasers fabricated using a single lithography step," Photonics Tech. Lett. 9, pp. 551, 1997.

[17] R. Thornton, Y. Zou, J. Tramontana, M. H. Crawford, R. P. Schneider, and K. D. Choquette. "Visible (670nm) vertical cavity surface emitting lasers with indium tin oxide transparent conducting top contacts," LEOS'95, San Francisco, CA, 1995 P.108.

[18] Wen-.Jang Jiang, Meng-Chyi Wu, Hsin-Chieh Yu, Chun-Yuan Huang, Chia-Pin Sung, Jim-Yong Chi, ”The 
effect of indium tin oxide as an ohmic contact for the $850 \mathrm{~nm}$ GaAs oxide-confined VCSELs," Solid-State Electron.46, pp. 1945, 2002. 